Jan Gaebel*, Johannes Keller, Daniel Schneider, Adrian Lindenmeyer, Thomas Neumuth, Stefan Franke

\title{
The Digital Twin: Modular Model-Based Approach to Personalized Medicine
}

\begin{abstract}
To overcome obstacles and complexity of decision making in clinical oncology, we propose an integrated clinical decision support approach; the Digital Twin. We analyse the reasons for frustration in applying clinical decision support and provide a multi-levelled approach to implementing a flexible system to support and strengthen clinical decisions. Describing medical patterns and contexts with Resource Description Framework (RDF) allows for standardised way of connecting medical knowledge and processing modules. Having flexible web-based interfaces integrated a multitude of heterogeneous data processing systems to either make clinical data available altogether, or provide calculations and assessments. Transition of the Digital Twin to clinical practice promises effective assistance and safer clinical decisions.
\end{abstract}

Keywords: Oncology; Therapy Decision Support; Decision Support System; Clinical Practice Guideline; Digital Technology; Computer-Assisted Image Processing, HL7 FHIR, Resource Description Framework

https://doi.org/10.1515/cdbme-2021-2057

\section{Introduction}

Clinical decision-making is a complex task with heterogeneous requirements depending on the medical subject. It involves experts from different domains and uses various tools. Large amounts of fragmented patient data, a multitude of clinical guidelines and a vast number of clinical studies need to be considered [1]. Furthermore, each physician in the course of clinical decision-making integrates clinical information into

\footnotetext{
*Jan Gaebel: Innovation Center Computer Assisted Surgery (ICCAS), Institut der Medizinischen Fakultät, Universität Leipzig, Semmelweisstr. 14, 04103 Leipzig, Germany,

jan.gaebel@medizin.uni-leipzig.de

Johannes Keller, Daniel Schneider, Adrian Lindenmeyer, Thomas Neumuth, Stefan Franke: Innovation Center Computer Assisted Surgery (ICCAS), Institut der Medizinischen Fakultät, Universität Leipzig, Leipzig, Germany
}

a mental model. Yet, usually they are left on their own devices. Altogether, this leads to a series of problems such as incomplete information due to only partial access to data and limited temporal as well as cognitive facilities, biased models due to the different background and interest of each expert, and obviously, multiple different models that need to be communicated and interrelated in expert meetings.

The most critical problem in therapeutic decision-making is the weighting of therapy options under consideration of the respective hypothetical patient outcome and the respective mental patient model. Clinical decision support has been a research topic for the last years. Several studies show promising results, in either medical accuracy or technical sophistication. However, these systems might not reach translation into clinical practice and provide limited value [2]. One of the many reasons slowing down development and acceptance is that medical information is often scarce or not directly usable by clinical decision support systems (CDSS). This also causes difficulties in utilizing machine-learning algorithms, which require large amounts of structured and annotated medical data. Making the limited data usable requires specific tools that need to be developed and each come with their own set of difficulties or requires time consuming manual labour which might fall to the medical staff. Pre-processing steps might include natural language processing to extract certain clinical items or concepts from clinical documents, and image processing to segment structures and extract information from them. Lastly, these systems must be quickly adaptable to new clinical knowledge or possibilities. Procedural changes in therapy must be integrated into existing models, as well as generally new emerging therapies, e.g., targeted therapies of precision medicine [3], should be recognized by the model.

In this paper, we address the above-mentioned problems by introducing the intentions of the BMBF-funded research project "Models for Personalized Medicine" (MPM) and present a universal approach to building a predictive diseaseand patient-specific therapeutic decision support system. We propose a framework that allows the aggregation and integration of multiple heterogeneous data sources and provide 
its combined and distributed knowledge to a modular network of specialised CDSS-modules.

First, we describe techniques for building a model that represents causal relations, patient information aggregated from medical health records, and knowledge derived from medical textbooks, clinical studies, and therapeutic guidelines - a Digital Twin. Although recently discussed as a general strategy [4], approaches of implementations of Digital Twins have been mostly application-specific, as for instance presented in $[5,6,7]$, which tend to form more or less rigid structures fitted to one specific use-case. At the core of our Digital Twin, the model is implemented as an interlinked Resource Description Framework (RDF) graph, capable of connecting the aggregating data. We showcase this capability with data derived from heterogeneous sources, such as electronic health records (EHR) as well as medical images. Second, we present techniques to use the capabilities of the Digital Twin with a modular and dynamic system that, for instance, provides data preparation, data processing, calculations of medical scores or even evidence-based simulations. Finally, we elaborate on the integration of such a system into clinical practice and the provision of sophisticated application systems for clinical translation.

\section{Methods}

We aim to propose an approach towards Digital Twins in medicine that supports a large variety of (tailored) decision support applications along various clinical pathways. Hence, the explainability of results in clinical contexts, a proper representation and handling of uncertainties, and reliable maintenance and validation of the applied models need to be considered carefully.

The overall architecture proposed here implements a Digital Twin as a middleware, which extends the capabilities of clinical information systems and serves as a single point of access to patient-related data for decision support applications (see also Fig. 2). The Digital Twin aims to representing all relevant characteristics of a patient and combining all the physicians' mental models which derive from their domain expert knowledge, experiences and assessments. It is supposed to provide a realistic depiction of the disease and all causal relations (e.g., pathophysiological properties). As a suitable tool to reach an extendible representation of multimodal clinical knowledge, RDF was chosen. Constructed as an RDF graph, we are able to reference patient data, represent information abstracted or projected from these data (e.g., ability to undergo general anaesthesia, tumour growth prediction), their interlinkage, and relations to documents of clinical knowledge (e.g., guidelines and studies).

Our first goal is to integrate routinely recorded patientspecific data into an RDF graph. In clinical practice, patient data is fragmented among multiple information systems using different data representations, as well as different ontologies to describe data. The Fast Healthcare Interoperability Resources (FHIR) standard [8,9] is an emerging data standard to represent, standardize and exchange primary health care data. We propose to translate existing patient data into valid FHIR resources. We expect that in the near future, most hospital information systems (HIS) will be able to serve their data in FHIR representation, so manually transcribing the data beforehand becomes obsolete. The FHIR standard also defines an RDF representation for its resources with a pre-defined namespace and vocabulary. We use this representation to integrate FHIR resources into the Digital Twin RDF graph. RDF data can be queried using the SPARQL query language [10]. As SPARQL queries tend to get quite complicated and are not very well adopted, the system additionally provides a GraphQL endpoint, making direct knowledge of RDF and SPARQL optional for external users of the system.

Chaining together different RDF descriptors, each representing an individual clinical entity, creates a network of medical knowledge and causal relations of one specific clinical use-case. Every node in this network fulfils a purpose. It can be a single patient-specific information entity taken from the hospital information system. Or, it can be a trigger for a specific processing of other nodes' data. These nodes are represented by clinical decision modules, that implement a solution to respective clinical question. Fig. 1 illustrates the $\mathrm{RDF}$ descriptor of an exemplary module for the calculation of the tumour size.

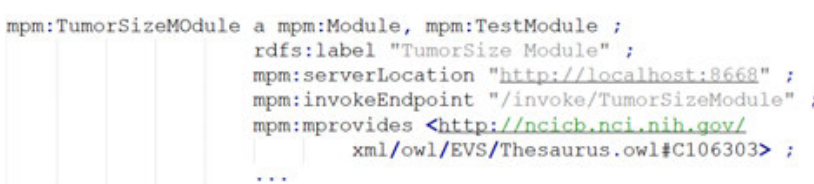

Figure 1: Exemplary definition of a module that provides a tumor size calculation described with a thesaurus concept from the National Cancer Institute.

There are several possible ways to implement these decision modules. From the technical point of view, there is no limitation (e.g., on what type of programming language is to be used). The only requirements are, that the modules implement a web interface that communicate clinical data using the systems intern data structure (to ensure the semantics and processible data), and that they provide their services live and in a timely manner (depending on the clinical question). 
They can realise specific novel solutions for a specific medical circumstance, or they can use already existing decision support systems that may yet be forming a more isolated application, e.g., data pre-processing for clinical decision support as described in [11].

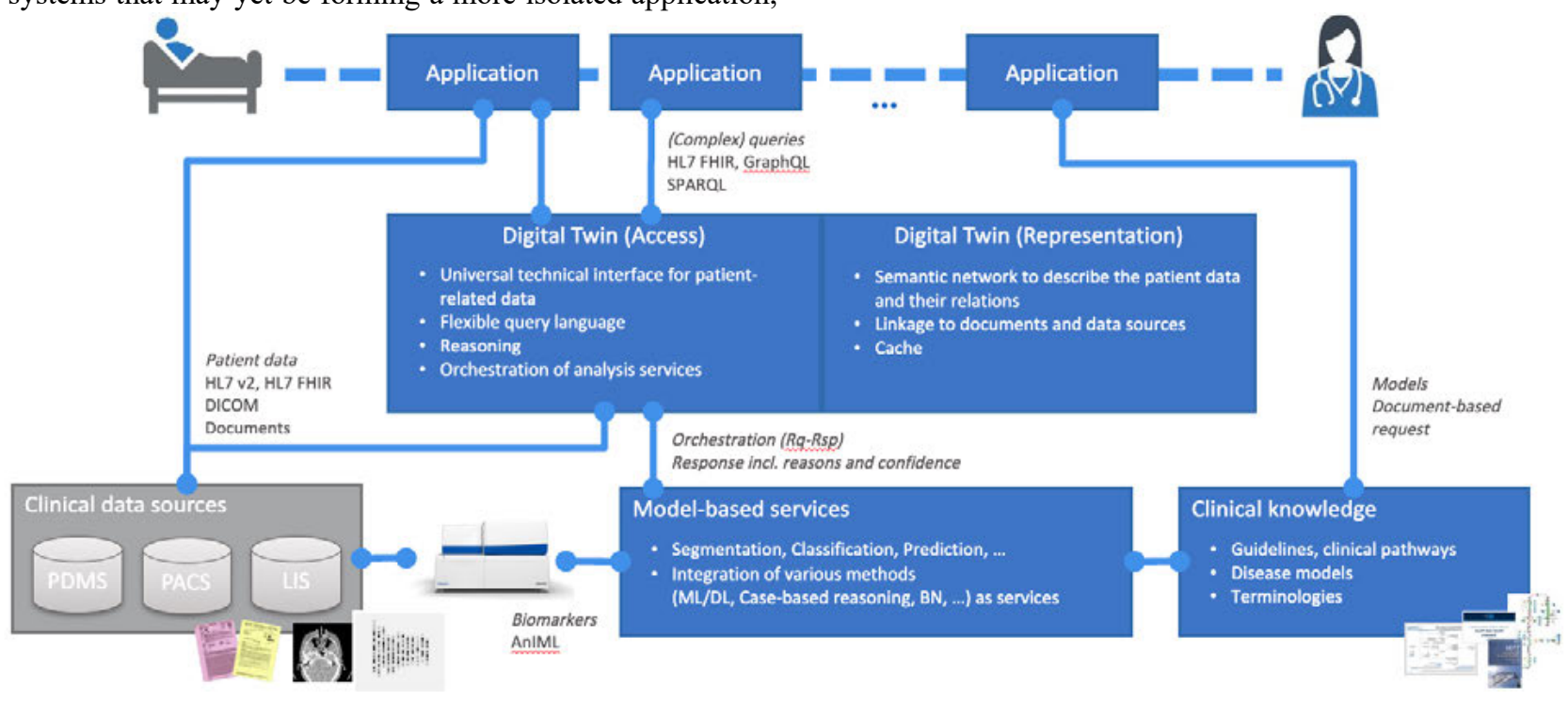

Figure 2: The architecture of the Digital Twin: located at the center, the Digital Twin stores its representative semantic network and controls the access and requests from clinical application systems as well as the data flow to decision modules or clinical knowledge bases.

If one module requires a specific input that is not available in the electronic patient record, these data can be provided by other modules. Decision modules can then invoke each other to create an even more sophisticated network of clinical knowledge and processing potential. Registered within the Digital Twin using a detailed RDF description, the Digital Twin access system can orchestrate the chain of decision modules and providing them with the knowledge of what functionalities are available. Theoretically, decision modules can be distributed over different medical expert networks. This enables the Digital Twin to utilize the most current and accurate knowledge without the restriction to local applications or libraries.

\section{Results}

The unified patient data representation of the Digital Twin enables the application of data-driven decision support. Accordingly, the Digital Twin is complemented by various rule- or machine learning-based modules providing derived information for data pruning and summarization, likelihood estimation and forecast, as well as anomaly detection, as illustrated in Fig.2. The tasks of these modules range from automatic radiomics generation, over data error detection to therapy advice and outcome prediction. All decision support routines are supplemented with instance-wise confidence estimations. Additionally, the hierarchical modular approach enables comprehensibility and human-readable explanations. This allows for maximum transparency and trustfulness.

Modules are connected via web services, exchanging their data (e.g., requesting a value or responding with a respective calculation) in JSON format. This network enables the integration of features from different, possibly remotely located expert systems and does not constrain the Digital Twin to one institution.

Clinical applications of the Digital Twin are viable for many different clinical questions or medical specialty. For instance, multidisciplinary team meetings for otolaryngological cancer patients are supported by preparing cases with plausibility checks from the Digital Twin. A patient's data can be processed to provide an assessment of his/her real situation regarding the tumour size (from an image processing module) or treatment feasibility from crosschecking the patient's pre-existing conditions based on safety scores. Neurosurgical follow-up treatment can be supervised with modules for early detection of tumour recurrence by evaluating treatment compliance with results from case-based reasoning.

A utilisation for a specific clinical setting can be implemented along the existing clinical pathways, either 
integrated in existing information systems or implemented as novel clinical user interfaces, e.g., provided through a web browser or mobile apps to increase accessibility. This ensures easy integration with relatively little additional manual labour to users, medical relevance from integration of patient-specific data and close alignment to clinical processes, and ultimately, high acceptance and trust from the clinical specialists.

\section{Discussion \& Conclusion}

We proposed the Digital Twin, as a computable representation of a patient with all relevant characteristics, based on an interlinked RDF graph capable of depicting causal relationships between different entities and describing processing pipelines. It can easily integrate and aggregate data from multiple sources such as EHR and knowledge derived from medical textbooks, clinical studies, and therapeutic guidelines. Surrounding the central Digital Twin, there can be a network of specialised service-oriented modules. This unifies the available data and provides a path for the interdisciplinary knowledge integration. As a result, this clinical data processing as a service provides a framework for the development and integration of future CDSS e.g., data preparation, calculations of medical scores or even evidencebased simulations and provide functionality to physicians and other healthcare professionals.

Nevertheless, we acknowledge that such a system itself requires maintenance that might not otherwise be present when developing a highly specialised CDSS system. Specialised data sources from emerging therapies need to be included into the Digital Twin first before they can be accessed. This requires the implementation of an RDF description of the data source and the new module itself, implementation of data transformations but also communicating the newly available data source to existing modules should it be beneficial. Negligence of due diligence in this regard might again result in fragmented knowledge and access to data.

The clinical domain is understandably fragmented due to many different professions and domains interacting, each with their own requirements and data sources. This might lead to many redundancies with many modules only providing slightly different information entities. Such redundancies together with the interlinking characteristic of the system can further complicate the maintenance overhead.
However, we argue that the benefits (reusability, automatic distribution and structuring of knowledge) outweigh the maintenance overhead.

\section{Author Statement}

Research funding: This project is funded by the German Federal Ministry of Education and Research (BMBF) grant number 03Z1L512. The statements made herein are solely the responsibility of the authors.

Conflict of interest: Authors state no conflict of interest.

\section{References}

[1] van Wijk Y, Halilaj I, van Limbergen E, Walsh S, Lutgens L, Lambin P, et al. Decision Support Systems in Prostate Cancer Treatment: An Overview. BioMed Research International. 2019 Jun 6;2019:1-10.

[2] Rockne RC, Hawkins-Daarud A, Swanson KR, Sluka JP, Glazier JA, Macklin P, et al. The 2019 mathematical oncology roadmap. Phys Biol. 2019 Jun 19;16(4):041005.

[3] Nussinov R, Jang H, Tsai C-J, Cheng F. Review: Precision medicine and driver mutations: Computational methods, functional assays and conformational principles for interpreting cancer drivers. Panchenko ARR, editor. PLoS Comput Biol. 2019 Mar 28;15(3):e1006658.

[4] Ahmed H, Devoto L. The Potential of a Digital Twin in Surgery. Surgical Innovation. December 2020. doi:10.1177/1553350620975896

[5] Chakshu NK, Sazonov I, Nithiarasu P. Towards enabling a cardiovascular digital twin for human systemic circulation using inverse analysis. Biomech Model Mechanobiol. 2021;20(2):449-465. doi:10.1007/s10237-020-01393-6

[6] Björnsson B, Borrebaeck C, Elander N, et al. Digital twins to personalize medicine. Genome Med. 2019;12(1):4. Published 2019 Dec 31. doi:10.1186/s13073-019-0701-3

[7] Corral-Acero J, Margara F, Marciniak M, et al. The 'Digital Twin' to enable the vision of precision cardiology. Eur Heart J. 2020;41(48):4556-4564. doi:10.1093/eurheartj/ehaa159

[8] Saripalle R, Runyan C, Russell M. Using HL7 FHIR to achieve interoperability in patient health record. J Biomed Inform. 2019;94:103188. doi:10.1016/j.jbi.2019.103188

[9] Lehne M, Luijten S, Vom Felde Genannt Imbusch P, Thun S. The Use of FHIR in Digital Health - A Review of the Scientific Literature. Stud Health Technol Inform. 2019;267:52-58. doi:10.3233/SHTI190805

[10] Lee J, Pham MD, Lee J, et al. Processing SPARQL queries with regular expressions in RDF databases. BMC Bioinformatics. 2011;12 Suppl 2(Suppl 2):S6. Published 2011 Mar 29. doi:10.1186/1471-2105-12-S2-S6

[11] Gaebel J, Wu H-G, Oeser A, et al. Modeling and Processing Up-To-Dateness of Patient Information in Probabilistic Therapy Decision Support. Artificial Intelligence in Medicine. Published online March 2020:101842. doi:10.1016/j.artmed.2020.101842 